\title{
STEREOLOGICAL CORRECTION OF MINERAL LIBERATION GRADE DISTRIBUTIONS ESTIMATED BY SINGLE SECTIONING OF PARTICLES
}

\author{
STEVEN SPENCER AND DAVID SUTHERLAND \\ CSIRO Minerals, PO Box 883, Kenmore QLD 4069, Australia \\ e-mail: steven.spencer@minerals.csiro.au \\ (Accepted August 3, 2000)
}

\begin{abstract}
The liberation distribution of ore samples is of considerable interest for process optimisation in the minerals industry. A scanning electron microscope-based automatic mineral analyser such as the LEO QEMSCAN system developed by CSIRO Minerals is a powerful tool for the estimation of linear or areal grade distributions of a population of ore particles based upon polished single particle sections. Stereological correction of a single section mineralogical grade distribution is recognised as an ill-posed inverse problem. The transformation kernel method with constrained entropy regularisation (King and Schneider, 1998) is adopted for the correction of stereological error in binary systems. An enhanced transformation kernel correction scheme is developed with an additional equality constraint for average grade as determined by section and volumetric sampling, in accordance with Delesse's fundamental stereological theorem. The usefulness of both correction methods is limited by the availability of kernels that appropriately model the relationship between volumetric and section grade distributions for the mineralogical sample of interest. The transformation kernel stereological correction methods are implemented in software available for use as part of the LEO QEMSCAN system. Both correction procedures are applied to areal section grade distributions of feed and concentrate from a mineral processing plant. The corrected grade distributions are in some instances found to be sensitive to the application of the average grade constraint. The statistical significance of differences in the corrected solutions is discussed.
\end{abstract}

Keywords: inverse problems, mineral liberation, stereology.

\section{INTRODUCTION}

Optimisation of mineral recovery processes requires accurate knowledge of the distribution of valuable minerals within comminuted (broken) ore particles. Transgranular fracture across mineral grains is dominant during comminution of many ores. Insufficient ore particle size reduction leads to a product with most valuable minerals remaining locked together with a significant amount of gangue (waste) material in composite particles. This may represent a substantial economic or technical problem for further beneficiation. Excessive grinding can result in liberated (single-phase) mineral particles that are too small to be subsequently efficiently separated from the gangue material by conventional methods, especially flotation.

Mineralogical analysis of ore and plant stream samples performed by scanning electron microscopy, uses a sample of order $10-1000 \mu \mathrm{m}$ particles of narrow size range that are mounted in a resin block. The LEO QEMSCAN system developed by CSIRO Minerals (Miller et al., 1982; Reid et al., 1984) identifies minerals by analysis of energy dispersive $\mathrm{x}$ ray (EDX) spectrum and backscattered electron intensity (BEI) from polished single sections of particles. Liberation measurements estimate the volumetric grade distribution of a mineral as a measure of the quality in a processing stream. This is the fraction of the total number of particles that contain a fraction, by volume, in a prescribed range (grade class) of the mineral of interest.

Stereological bias occurs in the 'unfolding' of the volumetric mineral liberation distribution of a sample of mineral particles from lower-dimensional section image analysis. Fig. 1 schematically illustrates the stereological bias problem for a two-phase mineralogical system. A section through a composite 
particle of simple texture has a finite probability of sampling only one species, leading to a systematic over-estimation of the proportion of mineral liberation. The extent of stereological bias depends on the texture of the ore, with samples containing mineral grains of size comparable to the particle exhibiting the most bias. Fig. 2 demonstrates the variety of mineralogical texture associated with a sample of particle areal sections considered in this study. When many of the particles are composite and the texture is simple (as shown in Fig. 2), stereological bias is an important consideration.
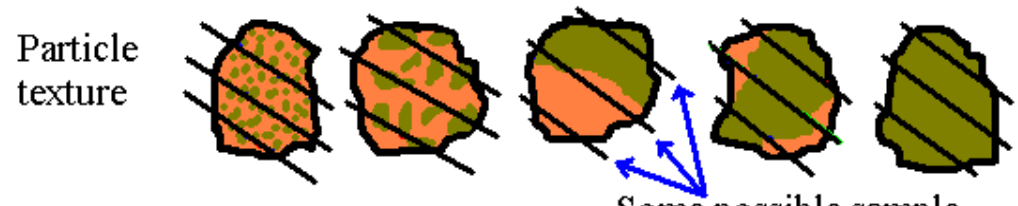

Some possible sample

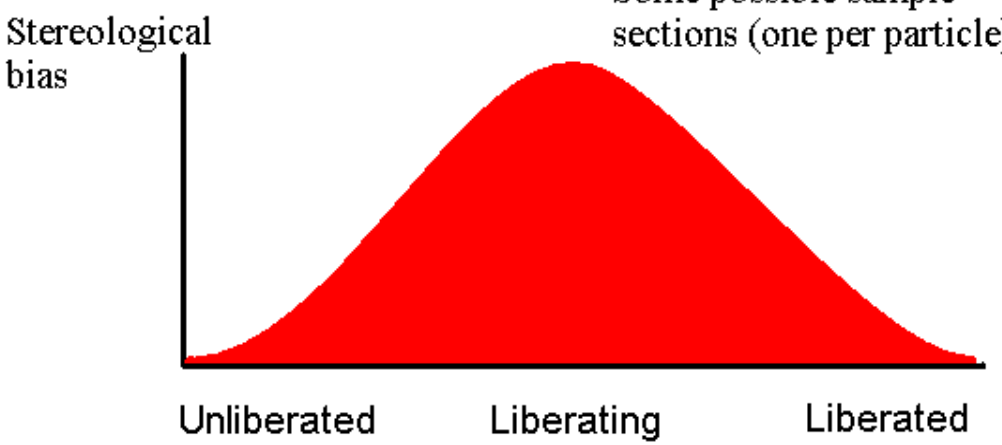

Fig. 1. Schematic of stereological error in grade estimation by single sectioning of particles. The magnitude of stereological bias is shown as a function of particle texture, with sections (straight lines) through liberated and composite particles of similar size.

Serial sections of particles can, in principle, overcome the problems associated with stereological error. However, the time, cost and complexity of accurate mineralogical volumetric phase estimation by multiple sections of each particle would be prohibitive in the context of automated image analysis with large numbers of particles and samples. Hence correction of stereological error related to grade estimation using single sections of particles is an important undertaking for quantitative analysis of mineralogical samples.

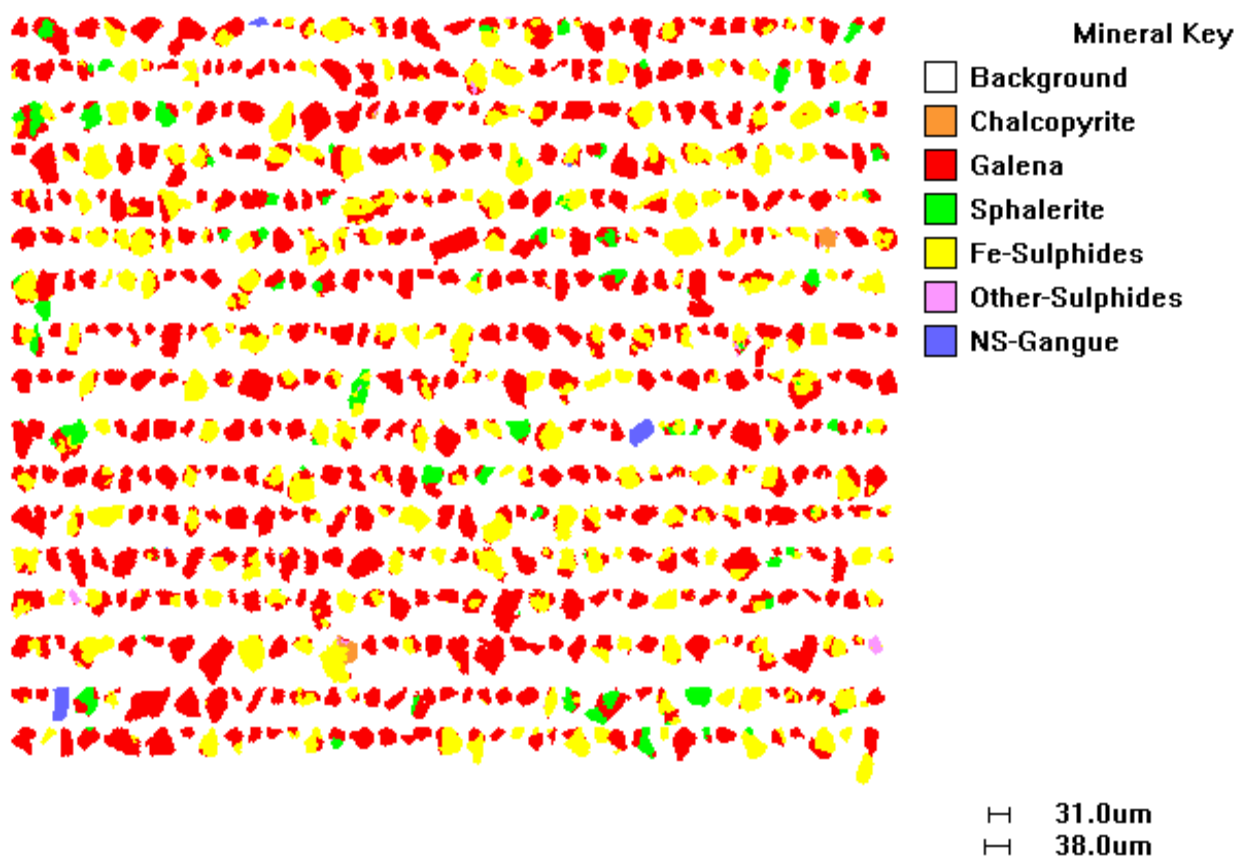

Fig. 2. Areal sections of a mineralogical sample from the final concentrate stream of an Australian lead/zinc mineral processing plant. 
Correction of mineralogical grade distributions for stereological bias can be classed as an ill-posed problem because very different volumetric grade solutions can be associated with quite similar apparent grade distributions (Groetsch, 1993; King and Schneider, 1998). Regularisation (stabilisation/ smoothing) constraints should be embedded in an algorithm that seeks to obtain the volumetric grade distribution from lower dimensional data in order to ensure the selection of the most physically reasonable solution (Groetsch, 1993; King and Schneider, 1998).

There are a number of stereological correction methods available for mineralogical samples modelled as binary systems. The parametric geometric probability method (Barbery, 1991; Leigh et al., 1996) couples statistical models for particle shape and mineral texture based on the assumption that breakage of particles occurs independently of any textural features (not generally the case in mineral processing) with measurements of sample averages for section grade, particle and mineral phase area or linear intercept length. The allocation method (Gay, 1995; Gay and Lyman, 1995) does not contain textural or breakage assumptions but rather attempts to estimate the sample volumetric grade distribution via grade class allocation of each measured section based on minimisation of an error function associated with satisfying a series of geometric probability equations for various volume weighted moments of particle grade. There are several variants of the latter scheme based on estimation of a kernel matrix relating apparent and volumetric grade distributions from stereological moment equations (Keith, 1997; Leigh et $a l .$, 1999). The main difficulty with these approaches is the underlying question of the statistical significance of the differences between the various weighted estimates of average grade used in the geometric probability equations.

The transformation kernel method (King, 1982; Schneider, 1995; King and Schneider, 1998; Fandrich et al., 1998) uses a constrained minimisation scheme to solve an ill-conditioned matrix inversion problem associated with estimating the true from the apparent liberation distribution. A texture-related kernel matrix which describes the linkage between grade class discretised volumetric and apparent grade distributions is selected from a library of experimental (Schneider, 1995; King and Schneider, 1998; Fandrich et al., 1998) and PARGEN (PARticle GENeration) synthetic kernels (Sepulveda et al., 1985) on the basis of providing the optimal fit of estimated true to apparent grade distribution. The current paucity of experimentally determined kernel matrices for a wide variety of mineralogical textures is seen as a limitation on the value of the scheme to industry (Fandrich et al., 1998).

The inversion technique utilised by the transformation kernel method incorporates constraints that are physically appropriate for any grade distribution. However, a fundamental stereological theorem (Delesse, 1847) states that the average grade as determined by point, line or area counting should be equal to the average volumetric grade in the limit of a large number of sections. Some of the metallurgical implications of applying the transformation kernel method constrained by Delesse's theorem to mineral processing plant liberation data have been discussed (Spencer and Sutherland, 2000). In this work, the effects of incorporating Delesse's theorem as an additional constraint specific to the sample into the transformation kernel method, including the statistical significance of differences in results, are investigated.

\section{METHOD}

The transformation kernel stereological correction equation relating observed particle section apparent to unknown volumetric grade distribution is expressed for a finite particle size interval as

$$
F_{a p p}\left\langle g_{a p p} \mid D\right\rangle=\int_{0}^{1} K\left\langle g_{a p p} \mid g_{v}, D\right\rangle f\left\langle g_{v} \mid D\right\rangle d g_{v},
$$

where:

$F_{a p p}\left\langle g_{\text {app }} \mid D\right\rangle$ is the measured cumulative fractional linear or areal distribution (weighted respectively by length or area according to the image analysis technique);

$g_{\text {app }}$ is the apparent grade;

$g_{v}$ is the volumetric grade;

$f\left\langle g_{v} \mid D\right\rangle$ is the unknown fractional volumetric grade distribution (weighted by volume);

$K\left\langle g_{\text {app }} \mid g_{v}, D\right\rangle$ is a kernel function representing the texture dependent relationship between apparent and volumetric grade (cumulative apparent grade distribution weighted by length or area, conditional on $g_{v}$ ) for a particle of characteristic size $D$ over a narrow size interval (King, 1982; Schneider, 1995; King and Schneider, 1998; Fandrich et al., 1998). 
Eq. 1 is a Fredholm integral equation of the first kind which is well-known to be ill-posed in terms of existence, uniqueness and stability of solution of the inversion problem (Groetsch, 1993). The transformation kernel stereological correction equation is expressed in grade class discretised form (with appropriately weighted functions) as

$$
\underline{F_{a p p}}=\underline{\underline{K}} \underline{f_{v}},
$$

where:

$F_{a p p} \quad$ is a vector of measured cumulative fractional distribution of apparent grades;

$f_{v} \quad$ is a vector of the unknown volumetric grade distribution for each grade class;

$K \quad$ is a kernel matrix representing the cumulative distribution of linear or areal grades as a function of volumetric grade for a particular narrow size range (King, 1982; Schneider, 1995; King and Schneider, 1998; Fandrich et al., 1998).

The ill-posed nature of the stereological correction problem, manifested by an ill-conditioned (high condition number) kernel matrix, means that attempts to directly invert Eq. 2 usually result in a non-physical solution. However, Eq. 2 can be stably inverted by use of the maximum entropy method as part of a constrained minimisation scheme (Groetsch, 1993; King and Schneider, 1998). The transformation kernel method with an augmented least-squares minimisation objective function based on the maximum entropy regularisation method can be written as

$$
\left\|100\left(\underline{\underline{K}} \underline{f_{v}}-\underline{F_{a p p}}\right)\right\|+\lambda \sum_{i=1}^{12} f_{v}^{i} \ln f_{v}^{i},
$$

where $f_{v}^{i}$ are the grade class components of the volumetric grade distribution (King and Schneider, 1998).

The physicality constraints on the unknown volumetric grade fractional distribution (usually in twelve classes) are

$$
\sum_{i=1}^{12} f_{v}^{i}=1
$$

and

$$
0 \leq f_{v}^{i} \leq 1
$$

The parameter $\lambda$ in Eq. 3 controls the amount of regularisation (smoothing) by maximisation of the solution entropy function $\left(-\sum_{i=1}^{12} f_{v}^{i} \ln f_{v}^{i}\right)$ relative to minimisation of the residual error norm $\left(\left\|100\left(\underline{\underline{K}} \underline{f_{v}}-\underline{F_{a p p}}\right)\right\|\right)$. For any given kernel function the optimal regularisation parameter maximises the entropy (measure of disorder or likelihood) of the volumetric grade distribution subject to minimising the solution error norm and maintaining other constraints, resulting in the most likely solution to the problem (Amato and Hughes, 1991; Hansen, 1992; Groetsch, 1993). L-curve analysis (so-called because of the shape of the curves - see Fig. 3) selects the optimal regularisation parameter and associated volumetric grade solution as the point of unity slope (usually $\lambda=1$ ) on the plot of solution entropy against residual norm. This solution represents the best balance between increasing the solution entropy of the solution and decreasing the residual error norm associated with the transformation (Hansen, 1992; King and Schneider, 1998).

Fig. 3 shows typical L-curves associated with stereological correction of single section mineral liberation distributions. Each curve in Fig. 3 is for a different kernel matrix (eight kernels were tested in this case) and data points on each curve represent the error norm and entropy associated with volumetric grade solutions obtained for different values of the entropy regularisation parameter. L-curve analysis is also used to select the most appropriate kernel to approximate the texture of the sample (see King and Schneider, 1998; Spencer and Sutherland, 2000). Kernels that are inappropriate to the texture of a sample result in a large residual error norm. The optimal volumetric grade solution, in terms of both the most appropriate kernel matrix and entropy regularisation parameter, has the lowest residual norm at the unity slope point on the L-curve (see Fig. 3). In this case the L-curve analysis shows that the two kernel functions associated with the curves on the left of the graph (experimentally determined from studies of other mineralogical samples) are suitable approximations for modelling the texture of this particular sample. The L-curves associated with PARGEN synthetic kernels (right of the graph) have a much larger error norm and are therefore much less suited to modelling the texture of the sample. 


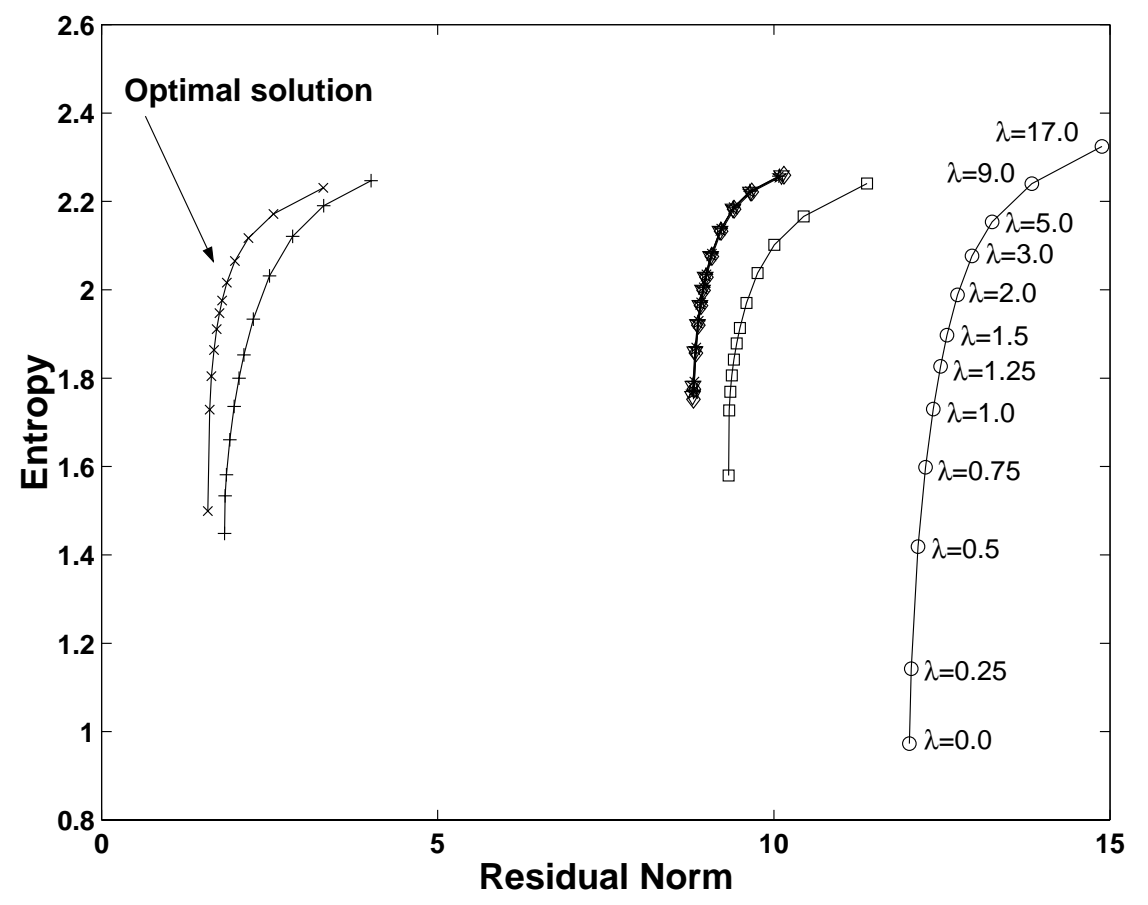

Fig. 3. Typical L-curve results for the constrained transformation kernel method. Optimal volumetric grade solution associated with the choice of kernel function and entropy regularisation parameter is as shown. Entropy regularisation parameter values are shown on the right for one kernel (same values for corresponding points on the other curves).

Delesse's average grade equality theorem can be written as

$$
E\left(g_{v}\right)=E\left(g_{a p p}\right),
$$

where: $E\left(g_{v}\right)$ and $E\left(g_{a p p}\right)$ are the expectations of respectively the volumetric (volume weighted) and apparent (line/area weighted) grade distributions.

It should be remembered that Delesse's theorem is only a stereological estimator for the first moment of the liberation distribution. Hence the need for the solution of the optimisation problem defined by Eqs. 3-5 with Eq. 6 taken to be an additional constraint. Ideally, it should also be ensured that the average apparent grade as determined by image analysis is within the experimental error of the (usually more accurate) estimate by chemical assay.

The average grade is estimated from the grade distribution both by direct application of Euler integration (trapezoidal rule) and by use of Simpson's rule on the cumulative grade distribution. This is done in order to establish the importance of discretisation error in the estimation of the average grade to the stereological correction process (King, 1999 - private communication). The constrained non-linear optimisa- tion problem for the optimal volumetric grade distribution as defined by Eqs. 3-6 is efficiently solved by the sequential quadratic programming method (Schittowski, 1985). The 'minimax' function of the Optimisation Toolbox in MATLAB is used in this study.

\section{RESULTS}

The LEO QEMSCAN system was used for liberation analysis of sphalerite (zinc mineral) samples from an Australian lead/zinc mineral processing plant. Fig. 4 shows typical comparisons of areal and stereologically corrected cumulative volumetric grade distributions (in mineral processing, the grade distribution is traditionally represented in cumulative form), derived from statistically valid samples of 1000 particle sections (Leigh et al., 1997). The corrected results are derived by the transformation kernel method (method A) and the same scheme coupled with the constraint of Delesse's theorem estimated by both Euler integration (method B) and Simpson's rule (method C). Fig. 4 shows $-75+53 \mu \mathrm{m}$ size class laboratory flotation cell feed and concentrate sphalerite grade distributions. There 
are clear differences between apparent and corrected grade distributions for both the feed and the concentrate, particularly in the stereologically expected tendency of apparent grade distributions to overestimate the fraction of fully liberated mineral and gangue particles. There is a decline in the estimated amount of pure gangue in the feed from an apparent value of $\sim 36 \%$ to $\sim 0-12 \%$, depending on the stereological correction technique. Similarly, the estimated amount of pure mineral in the concentrate declines from an apparent value of $\sim 18 \% \sim 0-9 \%$, depending on the stereological correction technique. Also, the feed sample apparent grade distribution in Fig. 4 indicates significant numbers of particles in every composite grade class. In contrast, the corrected grade distributions predict very little material in the
$20-70 \%$ grade range, indicating that much of the sphalerite has been liberated at the $-75+53 \mu \mathrm{m}$ grind size. These results are potentially important for the economic viability of the mineral processing.

Fig. 4 demonstrates clear differences between the results of stereological correction methods A, B and $\mathrm{C}$, particularly for the feed sample. There is a consistent difference of $\sim 4 \%$ in the cumulative grade predictions of method $\mathrm{A}$ and method $\mathrm{B}$ over most of the composite grade classes for the feed sample. However, the differences in the amount of pure liberated material predicted by stereological methods $\mathrm{A}, \mathrm{B}$ and $\mathrm{C}$ for both samples are relatively small in comparison to the difference between stereologically corrected and apparent values.

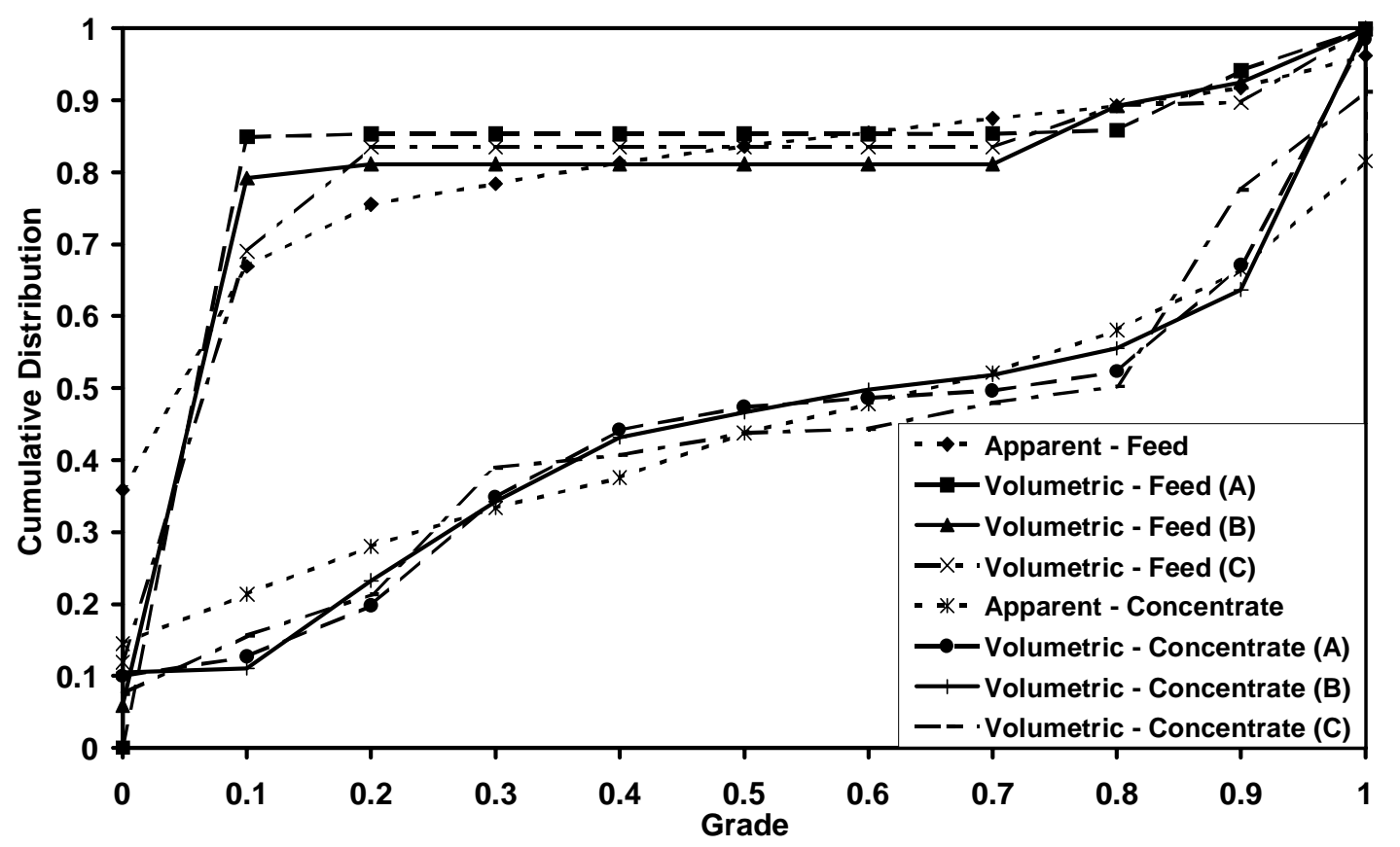

Fig. 4. Apparent (areal) and stereologically corrected (methods $A, B$ and $C$ ) sphalerite cumulative grade distributions for laboratory flotation cell feed and concentrate samples of $-75+53 \mu \mathrm{m}$ particles from an Australian lead/zinc mine.

Differences between the results of methods A, B and $\mathrm{C}$ do vary with sample. A bias associated with method A of underestimating the average volumetric grade (at larger error norms), generally increases with the error norm of the solution. The optimal sphalerite feed grade distribution estimated by method A has the largest error norm of the samples reported $(\sim 4.7 \%)$ and underestimates by $\sim 3 \%$ (absolute) the average grade of the sample. For the samples reported here, the differences between LEO QEMSCAN and chemical assay estimates of average volumetric grade are $<1 \%$, indicating that the number of particle sections analysed is representative of the parent population. Our experience with a variety of feed, concentrate and tail ore types from the processing streams of several mines has been that the imposition of Delesse's theorem as a constraint further stabilises the inversion procedure but usually increases the error norm associated with the solution. The latter could be interpreted as an indication that the 'true' kernel 
function representative of the texture of the particular sample diverges from the optimal kernel function selected from those available as the residual error norm of the solution increases.

Error estimates for the results of stereological correction procedures are not currently available. However, a useful approach is to consider the statistical significance of differences between apparent distributions and those corrected by various stereological procedures. The chi-squared $\left(\chi^{2}\right)$ test for two samples was applied to the binned differential grade distributions derived for this study. In terms of the number of sections sampled to determine the apparent grade distributions, all of the results (apparent, methods A, B and C) are significantly different for all of the samples. In the absence of a definitive scaling for the stereologically corrected data, ranking by the $\chi^{2}$-statistic of the differences between the various results is still seen as useful. This approach indicates that as expected, the most significant differences are between the apparent and the variously corrected grade distributions. The $\chi^{2}$ statistic differences between the results of methods B and $\mathrm{C}$ are of the same order as the differences between the method $\mathrm{A}$ and method $\mathrm{B} / \mathrm{C}$ sets of results. This result supports the view that the variation in the grade distribution induced by the change in the discretisation scheme for estimation of the average grade constraint is of the same order as the variation due to adding Delesse's theorem as a constraint on the transformation kernel approach. However for all cases investigated, the use of Simpson's rule rather than Euler integration to estimate the average grade resulted in a larger $\chi^{2}$-statistical difference to the transformation kernel method results unconstrained by average grade. The use of Delesse's theorem with Euler integration to estimate the average grade seems to be the most conservative approach.

\section{CONCLUSION}

Correction of stereological bias is essential to the accurate estimation of mineral liberation from single section samples. Liberation analyses of flotation feed and concentrate samples from an Australian lead/zinc mine show that single section apparent grade distributions can substantially over-estimate the proportion of pure liberated mineral of interest and gangue. Predictions of mineral processing plant performance based on such data could lead to serious technical and/or economic problems. The use of Delesse's theorem as an average grade constraint on the transformation kernel method of stereological correction is appropriate in order to ensure prediction of a volumetric grade distribution that is physically plausible for the sample under consideration. The stereologically corrected liberation results presented in this study in some instances show significant differences in predicted grade distribution due to the application of the average grade constraint. However, the choice of discretisation scheme for calculation of the average grade constraint can introduce changes to the corrected grade distribution of similar order to that caused by the imposition of the average grade constraint itself. Application of Delesse's theorem as an additional constraint in the correction process, with average grade determined by Euler integration, is recommended in cases where the best fit kernel results of the transformation kernel method produce a relatively large error norm and substantial error in estimated average grade.

The availability of a kernel matrix that accurately represents the texture of the sample currently under consideration is an important problem in the application of the transformation kernel approach to stereological correction of single section mineral liberation distributions. In the absence of a sufficient number of reliable stereological estimators to construct transformation kernels from single section information, the experimental determination of a broad range of kernel functions suitable for the large variety of textures likely to be encountered in mineral processing operations is an immediate concern.

A preliminary report of some of the data was presented at the $X^{\text {th }}$ International Congress for Stereology, Melbourne, Australia, 1-4 November 1999.

\section{REFERENCES}

Amato U, Hughes W (1991). Maximum entropy regularization of Fredholm integral equations of the first kind. Inverse Problems 7:793-808.

Barbery G (1991). Mineral Liberation: Measurement, Prediction and Use in Mineral Processing. Quebec: Les Editions GB.

Delesse MA (1847). Comptes rendus hebdomadaires des sciences de L'Academie de Sciences 25:544-5.

Fandrich RG, Schneider CL, Gay SL (1998). Two stereological correction methods:allocation method 
and kernel transformation method. Minerals Engineering 11:8:707-15.

Gay SL (1995). Stereological equations for phases within particles. J Microsc 179(3):297-305.

Gay SL, Lyman GJ (1995). Stereological error in particle sections-the solution. In: Proc. APCOM XXV Conf., Brisbane, Australia, 313-7.

Groetsch CW (1993). Inverse Problems in the Mathematical Sciences. Braunschweig/Wiesbaden: Friedr. Vieweg \& Son.

Hansen PC (1992). Analysis of discrete ill-posed problems by means of the L-curve. SIAM Review 34(4):561-80.

Keith JA (1997). New Method of Stereological Correction. In: The Sixth JKMRC Comference "Mine to Concentrator - A Modelling and Simulation Perspective", 75-84.

King RP (1982). The prediction of mineral liberation from mineralogical texture. In: Proc. XIV Int. Mineral Processing Congr. Toronto, Canada, 11-7.

King RP, Schneider CL (1998). Stereological correction of linear grade distributions for mineral liberation. Powder Technol 98:21-37.

Leigh GM, Hall P, Gay SL (1999). Nonparametric stereological methods for estimating mineral distribution. J Microsc, (submitted).

Leigh GM, Lyman GJ, Gay SL (1997). Computer-based examination of self-contained, flexible stereological correction procedures. Powder Technol 92:101-10.
Leigh GM, Lyman, GJ, Gottlieb P (1996). Stereological estimates of liberation from mineral section measurements: a rederivation of Barbery's formulae with extensions. Powder Technol 87:141-52.

Miller PR, Reid AF, Zuiderwyk MA (1982). QEM*SEM Image Analysis in the Determination of Modal Assays, Mineral Association and Mineral Liberation. In: Proc. XIV Int. Mineral Processing Congr., Toronto, Canada, VIII-3.

Reid AF, Gottlieb P, MacDonald KJ, Miller PR (1984). QEM*SEM Image Analysis of Ore Minerals: Volume Fraction, Liberation and Observational Variances. In: Park WC, Hausen DM, Hagni RD, eds. Applied Mineralogy. Warrendale, PA: The Metallurgical Society, 191-204.

Schittowski K (1985). NLQPL: A FORTRAN-Subroutine Solving Constrained Nonlinear Programming Problems. Ann Oper Res 5:485-500.

Schneider CL (1995). Measurement and calculation of liberation in continuous milling circuits. $\mathrm{PhD}$ thesis. University of Utah.

Sepulveda JE, Miller JD, Lin CL (1985). Generation of irregularly shaped multiphase particles for liberation analysis. In: Proc. XV Int. Mineral Processing Congr. Cannes, France 1:120-32.

Spencer SJ, Sutherland DN. Stereological correction of mineral liberation grade distributions. In: Proc. XXI Int. Mineral Processing Congr. Rome, Italy, 23-27 July 2000 (to appear). 